\title{
Multiple solutions to a perturbed Neumann problem
}

by

\section{Giuseppe Cordaro (Messina)}

Abstract. We consider the perturbed Neumann problem

$$
\begin{cases}-\Delta u+\alpha(x) u=\alpha(x) f(u)+\lambda g(x, u) & \text { a.e. in } \Omega, \\ \partial u / \partial \nu=0 & \text { on } \partial \Omega,\end{cases}
$$

where $\Omega$ is an open bounded set in $\mathbb{R}^{N}$ with boundary of class $C^{2}, \alpha \in L^{\infty}(\Omega)$ with $\operatorname{essinf}_{\Omega} \alpha>0, f: \mathbb{R} \rightarrow \mathbb{R}$ is a continuous function and $g: \Omega \times \mathbb{R} \rightarrow \mathbb{R}$, besides being a Carathéodory function, is such that, for some $p>N$, $\sup _{|s| \leq t}|g(\cdot, s)| \in L^{p}(\Omega)$ and $g(\cdot, t) \in L^{\infty}(\Omega)$ for all $t \in \mathbb{R}$. In this setting, supposing only that the set of global minima of the function $\frac{1}{2} \xi^{2}-\int_{0}^{\xi} f(t) d t$ has $M \geq 2$ bounded connected components, we prove that, for all $\lambda \in \mathbb{R}$ small enough, the above Neumann problem has at least $M+$ integer part of $M / 2$ distinct strong solutions in $W^{2, p}(\Omega)$.

1. Introduction. In this paper we consider the Neumann problem

$$
\begin{cases}-\Delta u+\alpha(x) u=\alpha(x) f(u)+\lambda g(x, u) & \text { a.e. in } \Omega \\ \partial u / \partial \nu=0 & \text { on } \partial \Omega\end{cases}
$$

where $\Omega \subset \mathbb{R}^{N}$ is a bounded and connected open set with boundary $\partial \Omega$ of class $C^{2}, \alpha \in L^{\infty}(\Omega)$ with $\operatorname{essinf}_{x \in \Omega} \alpha(x)>0, f: \mathbb{R} \rightarrow \mathbb{R}$ is a continuous function, $g: \Omega \times \mathbb{R} \rightarrow \mathbb{R}$ is a Carathéodory function, $\sup _{|s| \leq t}|g(\cdot, t)| \in L^{p}(\Omega)$ for some $p>N, g(\cdot, t) \in L^{\infty}(\Omega)$ for all $t \in \mathbb{R}$, and $\lambda$ is a real number.

A rather interesting question which arises in connection with a perturbation problem is to find conditions on $\phi$ and $\psi$ in order that there exists $\bar{\lambda}>0$ such that, for each $\lambda \in \mathbb{R}$ with $|\lambda| \leq \bar{\lambda}$, the boundary value problem

$$
\begin{cases}-\Delta u=\phi(x, u)+\lambda \psi(x, u) & \text { a.e. in } \Omega \\ \mathcal{B} u=0, & \text { on } \partial \Omega\end{cases}
$$

where $\mathcal{B}$ denotes the Dirichlet or Neumann boundary operator, admits at

2000 Mathematics Subject Classification: Primary 35J20.

Key words and phrases: Neumann problem, connected component, multiplicity of solutions, weak solution, strong solution. 
least $M$ distinct solutions whenever so does the unperturbed problem

$$
\begin{cases}-\Delta u=\phi(x, u) & \text { a.e. in } \Omega, \\ \mathcal{B} u=0 . & \text { on } \partial \Omega .\end{cases}
$$

Among the papers which treat the case of the Dirichlet problem (i.e. $\mathcal{B}=I$ ), we cite the most recent ones $[4,5]$. In both papers the unperturbed term $\phi$ is supposed to be odd with respect to the second variable while no growth conditions are imposed on the perturbation term $\psi$.

The case of the Neumann problem has been considered only recently, first in [9] and then in [2]. Also in these papers a feature is the absence of a growth condition on the perturbation term as in $[4,5]$, but the different nature of assumptions on $\phi$ is also worth noticing, in particular no symmetry properties are assumed. In both papers, variational methods are applied (see $[7,8]$ ). More precisely, the solutions of the problem are found to exist as local minima of the energy functional associated to problem $\left(P_{\lambda}\right)$. Our approach is similar since it is based on Theorem 2.1 of [1], which is a consequence of the general result stated in [7]. We focus on the same classes of nonlinearities $\phi$ (the unperturbed terms) as in [9]. Hence we devote the rest of this introduction to stating our result and comparing it with those cited above.

Set

$$
A_{f}=\left\{\xi \in \mathbb{R}: \frac{1}{2} \xi^{2}-\int_{0}^{\xi} f(t) d t=\inf _{t \in \mathbb{R}}\left(\frac{1}{2} t^{2}-\int_{0}^{t} f(s) d s\right)\right\} .
$$

Denote by $[r]$ the integer part of the real number $r$. Our result is as follows:

TheOREm 1.1. Suppose that the set $A_{f}$ has at least $M \geq 2$ bounded connected components in $\mathbb{R}$. Then there exist $\bar{a}, \bar{b} \in \mathbb{R}$ and $\bar{\lambda}, \sigma>0$ such that, for all $\lambda \in]-\bar{\lambda}, \bar{\lambda}\left[\right.$, problem $\left(P_{\lambda}\right)$ admits at least $M+[M / 2]$ distinct strong solutions in $W^{2, p}(\Omega), u_{i}^{(\lambda)}$ for $i=1, \ldots, M+[M / 2]$, satisfying $\left.u_{i}^{(\lambda)}(x) \in\right] \bar{a}, \bar{b}[$ for a.e. $x \in \Omega$, and $\int_{\Omega}\left|\nabla u_{i}^{(\lambda)}(x)\right|^{2} d x \leq \sigma$.

The key assumption of the above theorem is the existence of distinct connected components of the set $A_{f}$ in $\mathbb{R}$. This assumption has been used for the first time in [9]. For the reader's convenience, we recall that theorem:

Theorem A ([9, Theorem 1]). Let $f: \mathbb{R} \rightarrow \mathbb{R}$ be a continuous function such that

$$
\lim _{|\xi| \rightarrow \infty} \frac{f(\xi)}{\xi}=0 .
$$

Assume that the set $A_{f}$ has at least $M \geq 2$ connected components. Then, 
for every $\alpha \in L^{\infty}(\Omega)$ with $\operatorname{ess}_{\inf } \Omega>0$, for every

$$
\varrho>\|\alpha\|_{L}^{1}(\Omega) \inf _{\xi \in \mathbb{R}}\left(\frac{\xi^{2}}{2}-\int_{0}^{\xi} f(t) d t\right)
$$

and for every Carathéodory function $g: \Omega \times \mathbb{R} \rightarrow \mathbb{R}$ such that $\sup _{|\xi| \leq s}|g(\cdot, \xi)|$ $\in L^{p}(\Omega)$ for some $p>N$ and all $s>0$, there exists $\lambda^{*}>0$ such that, for each $\lambda \in] 0, \lambda^{*}\left[\right.$, the Neumann problem $\left(P_{\lambda}\right)$ admits at least $M+1$ strong solutions in $W^{2, p}(\Omega), M$ of which belong to the set

$$
\left\{u \in W^{2, p}(\Omega): \frac{1}{2} \int_{\Omega}|\nabla u(x)|^{2} d x+\int_{\Omega} \alpha(x)\left(\frac{|u(x)|^{2}}{2}-\int_{0}^{u(x)} f(t) d t\right) d x<\varrho\right\} .
$$

The sublinearity assumption (f) on the nonlinear term $f$ motivated some of our investigations. Here, in particular, we show that the requirement of sublinear behaviour for $f$ near $\infty$ can be dropped provided that the connected components of the set $A_{f}$ are bounded. Moreover, exploiting the fact that, in our setting, $g(\cdot, t) \in L^{\infty}(\Omega)$, we are able to prove the existence of at least $M+[M / 2]$ strong solutions to $\left(P_{\lambda}\right)$, for $\lambda$ small enough, when $A_{f}$ has $M$ connected components. So, in our setting, the number of solutions is greater than what it is ensured by Theorem A. This fact is worthy of being stressed in view of the result recently proved by Anello which we quote below:

Theorem B ([2, Theorem 2]). Let $\left[a_{1}, b_{1}\right], \ldots,\left[a_{M}, b_{M}\right]$ be $M$ compact pairwise disjoint real intervals, $D$ a set containing $\bigcup_{i=1}^{M}\left[a_{i}, b_{i}\right]$, and $f: D \rightarrow \mathbb{R}$ a continuous function satisfying

$$
\max \left\{F\left(a_{i}\right), F\left(b_{i}\right)\right\} \leq \max _{\xi \in\left[a_{i}, b_{i}\right]} F(\xi) \quad(i=1, \ldots, M),
$$

where $F(t)=\frac{1}{2} t^{2}-\int_{0}^{t} f(s) d s$. Then, for every Carathéodory function $g$ : $\Omega \times \mathbb{R} \rightarrow \mathbb{R}$ satisfying $\sup _{t \in D}|g(\cdot, t)| \in L^{p}(\Omega)$ for some $p>N$, there exist $\bar{\lambda}, \sigma>0$ such that, for every $\lambda \in[-\bar{\lambda}, \bar{\lambda}]$, there exist $M$ strong solutions $u_{1}^{\lambda}, \ldots, u_{M}^{(\lambda)} \in W^{2, p}(\Omega)$ of problem $\left(P_{\lambda}\right)$ satisfying $\left.u_{i}^{(\lambda)} \in\right] a_{i}, b_{i}[$ for a.e. $x \in \Omega$ and $i=1, \ldots, M$, and

$$
\max _{i \in\{1, \ldots, M\}} \int_{\Omega}\left(\left|\nabla u_{i}^{(\lambda)}\right|^{2}+\left|u_{i}^{(\lambda)}\right|^{2}\right) d x \leq \sigma .
$$

2. Proof of Theorem 1.1. For $i=1, \ldots, M$, denote by $\left[a_{i}, b_{i}\right]$ the bounded connected components such that

$$
A_{f} \supseteq \bigcup_{i=1}^{M}\left[a_{i}, b_{i}\right]
$$

and $a_{1} \leq b_{1}<a_{2} \leq b_{2}<\cdots<a_{M} \leq b_{M}$. It is easily seen that, for all $1 \leq i$ $\leq M$, there exist $c_{i}, d_{i} \in \mathbb{R}$ such that $\left[a_{i}, b_{i}\right] \subset\left[c_{i}, d_{i}\right],\left[c_{i}, d_{i}\right] \cap\left[c_{i+1}, d_{i+1}\right]=\emptyset$ 
and

$$
c_{i}<f\left(c_{i}\right), \quad d_{i}>f\left(d_{i}\right) .
$$

First, we prove that there exists $\lambda_{1}>0$ such that, for each $\left.\lambda \in\right]-\lambda_{1}, \lambda_{1}[$, problem $\left(P_{\lambda}\right)$ has at least three solutions with values in $\left[c_{1}, d_{2}\right]$.

We consider the truncations of $f$ and $g$ to $\left[c_{1}, d_{2}\right]$, defined as follows:

$$
f_{1}(t)=\left\{\begin{array}{ll}
f(t) & \text { if } c_{1} \leq t \leq d_{2}, \\
f\left(c_{1}\right) & \text { if } t<c_{1}, \\
f\left(d_{2}\right) & \text { if } t>d_{2}
\end{array} \quad g_{1}(x, t)= \begin{cases}g(x, t) & \text { if } c_{1} \leq t \leq d_{2}, \\
g\left(x, c_{1}\right) & \text { if } t<c_{1}, \\
g\left(x, d_{2}\right) & \text { if } t>d_{2} .\end{cases}\right.
$$

$W^{1,2}(\Omega)$ is endowed with the norm

$$
\|\cdot\|_{\alpha, W^{1,2}(\Omega)}=\left(\int_{\Omega}|\nabla(\cdot)|^{2}+\int_{\Omega} \alpha(x)|\cdot|^{2}\right)^{1 / 2}
$$

which is equivalent to the usual one. Then we define the functionals $\Psi$ and $\Phi$ on $W^{1,2}(\Omega)$ by

$$
\begin{aligned}
& \Psi(u)=\frac{1}{2}\|u\|_{\alpha, W^{1,2}(\Omega)}^{2}-\int_{\Omega}\left(\int_{0}^{u(x)} \alpha(x) f_{1}(t) d t\right) d x, \\
& \Phi(x)=-\int_{\Omega}\left(\int_{0}^{u(x)} g_{1}(x, t) d t\right) d x .
\end{aligned}
$$

It is well known that $\Psi$ and $\Phi$ are well defined, weakly sequentially lower semicontinuous and continuously differentiable on $W^{1,2}(\Omega)$. Moreover, a critical point of $\Psi+\lambda \Phi$ is a weak solution in $W^{1,2}(\Omega)$ of the Neumann problem

$$
\begin{cases}-\Delta u+\alpha(x) u=\alpha(x) f_{1}(u)+\lambda g_{1}(x, u) & \text { a.e. in } \Omega \\ \partial u / \partial \nu=0 & \text { on } \partial \Omega .\end{cases}
$$

Suppose that $w$ is a weak solution of $\left(P_{1, \lambda}\right)$. The function $\bar{w}: \Omega \rightarrow \mathbb{R}$ defined by

$$
\bar{w}(x)= \begin{cases}w(x) & \text { if } c_{1} \leq w(x) \leq d_{2} \\ c_{1} & \text { if } w(x)<c_{1} \\ d_{2} & \text { if } w(x)>d_{2}\end{cases}
$$

belongs to $W^{1,2}(\Omega)$. So, by exploiting the fact that $w$ is a weak solution of $\left(P_{1, \lambda}\right)$, one obtains

$$
\begin{aligned}
\int_{\Omega} \nabla w(x) \nabla(w(x)-\bar{w}(x)) d x & +\int_{\Omega} \alpha(x) w(x)(w(x)-\bar{w}(x)) d x \\
= & \int_{\Omega} \alpha(x) f_{1}(w(x))(w(x)-\bar{w}(x)) d x \\
& +\lambda \int_{\Omega} g_{1}(x, w(x))(w(x)-\bar{w}(x)) d x .
\end{aligned}
$$


Taking into account that

$$
\int_{\Omega} \nabla \bar{w}(x) \nabla(w(x)-\bar{w}(x)) d x=0,
$$

from (2.1) it follows that

$$
\begin{aligned}
& \int_{\Omega}|\nabla(w(x)-\bar{w}(x))|^{2} d x+\int_{\Omega} \alpha(x)(w(x)-\bar{w}(x))^{2} d x \\
& =\int_{\Omega} \alpha(x)\left(f_{1}(w(x))-\bar{w}(x)\right)(w(x)-\bar{w}(x)) d x \\
& \quad+\lambda \int_{\Omega} g_{1}(x, w(x))(w(x)-\bar{w}(x)) d x \\
& \leq \quad \int_{\left\{w<c_{1}\right\}}\left[(\operatorname{ess} \sup \alpha)\left(f\left(c_{1}\right)-c_{1}\right)+\lambda g\left(x, c_{1}\right)\right]\left(w(x)-c_{1}\right) d x \\
& \quad+\int_{\left\{w>d_{2}\right\}}\left[(\operatorname{essinf} \alpha)\left(f\left(d_{2}\right)-d_{2}\right)+\lambda g\left(x, d_{2}\right)\right]\left(w(x)-d_{2}\right) d x
\end{aligned}
$$

There exists $\lambda^{*}>0$ such that, for all $\left.\lambda \in\right]-\lambda^{*}, \lambda^{*}[$,

$$
(\underset{\Omega}{\operatorname{ess} \sup } \alpha)\left(f\left(c_{1}\right)-c_{1}\right)+\lambda g\left(x, c_{1}\right)>0
$$

and

$$
(\underset{\Omega}{\operatorname{essinf}} \alpha)\left(f\left(d_{2}\right)-d_{2}\right)+\lambda g\left(x, d_{2}\right)<0,
$$

for a.e. $x \in \Omega$. Hence, from (2.2), it follows that $w(x)=\bar{w}(x)$ for a.e. $x \in \Omega$. So every weak solution of $\left(P_{1, \lambda}\right)$ is a weak solution of $\left(P_{\lambda}\right)$ provided that $\lambda \in]-\lambda^{*}, \lambda^{*}[$.

Now, we note that the function $F_{1}: \mathbb{R} \rightarrow \mathbb{R}$ defined by

$$
F_{1}(t)=\frac{1}{2} t^{2}-\int_{0}^{t} f_{1}(s) d s
$$

reaches its global minimum at every point of the set $\left[a_{1}, b_{1}\right] \cup\left[a_{2}, b_{2}\right]$. Consequently, the constant functions

$$
w_{i}(x)=\frac{a_{i}+b_{i}}{2} \quad(x \in \Omega \text { and } i=1,2)
$$

turn out to be two distinct points of global minimum for the functional $\Psi$. Fix $r_{1}, r_{2} \in \mathbb{R}$ such that, for $i=1,2$,

$$
\begin{aligned}
& \frac{b_{i}-a_{i}}{2}\left(\int_{\Omega} \alpha(x) d x\right)^{1 / 2}<r_{i} \\
& \quad \leq\left[\min \left\{\left(d_{i}-b_{i}\right),\left(a_{i}-c_{i}\right)\right\}+\frac{b_{i}-a_{i}}{2}\right]\left(\int_{\Omega} \alpha(x) d x\right)^{1 / 2} .
\end{aligned}
$$


It follows that

$$
r_{1}+r_{2}<\left\|w_{1}-w_{2}\right\|_{\alpha, W^{1,2}(\Omega)} .
$$

At this point, in order to apply Theorem 2.1 of [1], it remains to show that, for $i=1,2$,

$$
\Psi\left(w_{i}\right)<\inf _{\|v\|_{\alpha, W^{1,2}(\Omega)}=r_{i}} \Psi\left(w_{i}+v\right) .
$$

Arguing by contradiction, suppose that

$$
\Psi\left(w_{i}\right)=\inf _{\|v\|_{\alpha, W^{1,2}(\Omega)}=r_{i}} \Psi\left(w_{i}+v\right) .
$$

Then there would exist a sequence $\left\{v_{n}\right\}$ with $\left\|v_{n}\right\|_{\alpha, W^{1,2}(\Omega)}=r_{i}$ for $n \in \mathbb{N}$ such that

$$
\begin{aligned}
\Psi\left(w_{i}\right)= & \lim _{n \rightarrow \infty} \Psi\left(w_{i}+v_{n}\right) \\
= & \lim _{n \rightarrow \infty} \frac{1}{2}\left(\left\|w_{i}\right\|_{\alpha, W^{1,2}(\Omega)}^{2}+\left\|v_{n}\right\|_{\alpha, W^{1,2}(\Omega)}^{2}\right) \\
& +\int_{\Omega} \alpha(x) w_{i}(x) v_{n}(x) d x-\int_{\Omega}\left(\int_{0}^{w_{i}(x)+v_{n}(x)} \alpha(x) f(t) d t\right) d x .
\end{aligned}
$$

Consequently, up to a subsequence, $v_{n} \rightarrow \bar{v}_{i}$ weakly in $W^{1,2}(\Omega)$ as $n \rightarrow \infty$, for some $\bar{v}_{i} \in W^{1,2}(\Omega)$ with $\left\|\bar{v}_{i}\right\|_{\alpha, W^{1,2}(\Omega)} \leq r_{i}$. Owing to the Rellich-Kondrashov compact embedding theorem for $W^{1,2}(\Omega)$, by (2.7), one has

$$
\begin{aligned}
\Psi\left(w_{i}\right)= & \frac{1}{2}\left\|w_{i}\right\|_{\alpha, W^{1,2}(\Omega)}^{2}+\frac{1}{2} r_{i}^{2}+\int_{\Omega} \alpha(x) w_{i}(x) \bar{v}_{i}(x) d x \\
& -\int_{\Omega}\left(\int_{0}^{w_{i}(x)+\bar{v}_{i}(x)} \alpha(x) f(t) d t\right) d x \\
\geq & \frac{1}{2} \int_{\Omega}\left|\nabla \bar{v}_{i}(x)\right|^{2} d x+\frac{1}{2} \int_{\Omega} \alpha(x)\left(w_{i}(x)+\bar{v}_{i}(x)\right)^{2} d x \\
& -\int_{\Omega}\left(\int_{0}^{w_{i}(x)+\bar{v}_{i}(x)} \alpha(x) f(t) d t\right) d x .
\end{aligned}
$$

Taking into account that $\Psi\left(w_{i}\right)$ is a global minimum for $\Psi$, from (2.8) it follows that

$$
\left|\nabla \bar{v}_{i}(x)\right|=0 \quad \text { for a.e. } x \in \Omega
$$

and

$$
\int_{\Omega} \alpha(x) \bar{v}_{i}^{2}(x) d x=r_{i}^{2} .
$$

By (2.8), $\bar{v}_{i}$ is constant, $\Omega$ being connected. 
Moreover, (2.8) also implies that

$$
w_{i}(x)+\bar{v}_{i}(x) \in\left[a_{1}, b_{1}\right] \cup\left[a_{2}, b_{2}\right]
$$

for a.e. $x \in \Omega$ and $i=1,2$. This leads to a contradiction in view of (2.9) and (2.3). So, (2.5) is proved.

By Theorem 2.1 of [1], there exists $\tilde{\lambda}>0$ such that, for each $\lambda \in]-\widetilde{\lambda}, \widetilde{\lambda}[$, $\Psi+\lambda \Phi$ has at least two local minima $u_{\lambda}^{(1)}, u_{\lambda}^{(2)}$ with $\left\|u_{\lambda}^{(i)}-w_{i}\right\|_{\alpha, W^{1,2}(\Omega)} \leq$ $r_{i}(i=1,2)$. Moreover, $\Psi+\lambda \Phi$ satisfies the Palais-Smale condition (see Example 38.25 in [12]), so Theorem 1 of [6] implies the existence of a third critical point $u_{\lambda}^{(3)}$ distinct from $u_{\lambda}^{(1)}$ and $u_{\lambda}^{(2)}$. Set $\lambda_{1}=\min \left\{\lambda^{*}, \tilde{\lambda}\right\}$. For each $\lambda \in]-\lambda_{1}, \lambda_{1}\left[, u_{\lambda}^{(1)}, u_{\lambda}^{(2)}, u_{\lambda}^{(3)}\right.$ are weak solutions to $\left(P_{\lambda}\right)$. Furthermore, for $|\lambda|<\lambda_{1}$ and $i=1,2$, one has

$$
\begin{aligned}
\int_{\Omega}\left|\nabla u_{i}^{(\lambda)}(x)\right|^{2} d x & \leq\left\|u_{i}^{(\lambda)}\right\|_{\alpha, W^{1,2}(\Omega)}^{2} \\
& \leq\left\|w_{i}\right\|_{\alpha, W^{1,2}(\Omega)}^{2}+\left\|u_{i}^{(\lambda)}-w_{i}\right\|_{\alpha, W^{1,2}(\Omega)}^{2} \leq \sigma_{i}^{2}
\end{aligned}
$$

where $\sigma_{i}$ does not depend on $\lambda$. Put $\bar{\sigma}=\max \left\{\sigma_{1}, \sigma_{2}\right\}$. Note that $u_{3}^{(\lambda)}$ satisfies

$$
\Psi\left(u_{3}^{(\lambda)}\right)+\lambda \Phi\left(u_{3}^{(\lambda)}\right)=c(\lambda),
$$

where

$$
c(\lambda)=\inf _{\psi \in \Gamma_{\lambda}} \sup _{t \in[0,1]}(\Psi(\psi(t))+\lambda \Phi(\psi(t)))
$$

and

$$
\Gamma_{\lambda}=\left\{\psi \in C([0,1], H): \psi(0)=u_{1}^{(\lambda)} \text { and } \psi(1)=u_{2}^{(\lambda)}\right\} .
$$

For every $\lambda \in]-\lambda_{1}, \lambda_{1}\left[\right.$, the function $\psi_{\lambda}: t \in[0,1] \mapsto u_{1}^{(\lambda)}+(1-t) u_{2}^{(\lambda)}$ belongs to $\Gamma_{\lambda}$. Moreover, by (2.10),

$$
\sup _{|\lambda|<\lambda_{1}} \sup _{t \in[0,1]}\left\|\psi_{\lambda}(t)\right\| \leq 2 \bar{\sigma} .
$$

So, exploiting the fact that $\Psi$ is the sum of the norm squared and a sequentially weakly continuous functional and that $\Phi$ is sequentially weakly continuous, one has

$$
\begin{aligned}
\sup _{|\lambda|<\lambda_{1}} \phi(\lambda) & \leq \sup _{|\lambda|<\lambda_{1}} \sup _{t \in[0,1]}\left(\Psi\left(\psi_{\lambda}(t)\right)+\lambda \Phi\left(\psi_{\lambda}(t)\right)\right) \\
& \leq \sup _{\|v\| \leq 2 \bar{\sigma}} \Psi(v)+\bar{\lambda} \sup _{\|v\| \leq 2 \bar{\sigma}} \Phi(v)<\infty .
\end{aligned}
$$

If

$$
\sup _{|\lambda|<\lambda_{1}}\left\|u_{3}^{(\lambda)}\right\|=\infty
$$


there would exist a bounded sequence $\left.\left\{\lambda_{n}\right\} \subset\right]-\lambda_{1}, \lambda_{1}\left[\right.$ such that $\left\|u_{3}^{\left(\lambda_{n}\right)}\right\|$ $\rightarrow \infty$ as $n \rightarrow \infty$. Consequently, we would have

$$
\begin{aligned}
\lim _{n \rightarrow \infty} \phi\left(\lambda_{n}\right) & =\lim _{n \rightarrow \infty}\left(\Psi\left(u_{3}^{\left(\lambda_{n}\right)}\right)+\lambda_{n} \Phi\left(u_{3}^{\left(\lambda_{n}\right)}\right)\right) \\
& \geq \lim _{n \rightarrow \infty}\left(\Psi\left(u_{3}^{\left(\lambda_{n}\right)}\right)+\lambda_{1} \min \left\{0, \Phi\left(u_{3}^{\left(\lambda_{n}\right)}\right)\right\}\right)=\infty,
\end{aligned}
$$

contrary to (2.11). In order to complete the proof, we can repeat the arguments used above for any distinct couple of intervals $\left[a_{i}, b_{i}\right]$ and $\left[a_{i+1}, b_{i+1}\right]$. Hence, for $i=1, \ldots,[M / 2]$, there exists $\lambda_{i}>0$ such that, for each $\lambda \in$ ]$-\lambda_{i}, \lambda_{i}\left[,\left(P_{\lambda}\right)\right.$ has three distinct solutions with values in $\left[c_{2 i-1}, d_{2 i}\right]$. If $M$ is odd, it is easy to prove that there exists $\lambda_{[M / 2]+1}>0$ such that, for $\lambda \in]-\lambda_{[M / 2]+1}, \lambda_{[M / 2]+1}\left[,\left(P_{\lambda}\right)\right.$ has a weak solution with values in $\left[c_{M}, d_{M}\right]$. Put $\bar{\lambda}=\min \left\{\lambda_{1}, \ldots, \lambda_{M / 2}\right\}$ if $M$ is even, and $\bar{\lambda}=\min \left\{\lambda_{1}, \ldots, \lambda_{[M / 2]+1}\right\}$ if $M$ is odd. The conclusion follows by taking into account that a weak solution $u_{i}^{(\lambda)}$ is a strong solution which belongs to $W^{2, p}(\Omega)$ because the right hand side $\alpha(\cdot) f\left(u_{i}^{\lambda}(\cdot)\right)+\lambda g\left(\cdot, u_{i}^{(\lambda)}(\cdot)\right)$ belongs to $L^{p}(\Omega)$ (see $[11,3,10]$ ).

3. A typical application. We now give an application of Theorem 1.1 to the problem considered in Proposition 1 of [9].

Let $\psi:\left[0, \infty\left[\rightarrow \mathbb{R}\right.\right.$ be a $C^{1}$ periodic function such that $a=\inf _{[0 \infty[} \psi$ $<\psi(0)$, and consider the Neumann problem

$$
\begin{cases}-\Delta u=\frac{\alpha(x) u}{2(\psi(0)-a)}\left(\frac{\gamma}{|u|^{\gamma}} \psi^{\prime}\left(\frac{1}{|u|^{\gamma}}\right)-2\left(\psi\left(\frac{1}{|u|^{\gamma}}\right)-a\right)\right)+ & \lambda g(x, u) \\ \partial u / \partial \nu=0 & \text { a.e. in } \Omega \\ \text { on } \partial \Omega\end{cases}
$$

where $\Omega, \alpha, g$ are as in the introduction and $\gamma \in] 0,1[$.

So, the function $f: \mathbb{R} \rightarrow \mathbb{R}$ is as follows:

$$
f(t)= \begin{cases}t+\frac{t}{2(\psi(0)-a)}\left(\frac{\gamma}{|t|^{\gamma}} \psi^{\prime}\left(\frac{1}{|t|^{\gamma}}\right)-2\left(\psi\left(\frac{1}{|t|^{\gamma}}\right)-a\right)\right) & \text { if } t \neq 0, \\ 0 & \text { if } t=0 .\end{cases}
$$

Since $\gamma \in] 0,1[, f$ is continuous. Moreover,

$$
\frac{\xi^{2}}{2}-\int_{0}^{\xi} f(t) d t= \begin{cases}\frac{\xi^{2}}{2(\psi(0)-a)}\left(\psi\left(\frac{1}{|\xi|^{\gamma}}\right)-a\right) & \text { if } \xi \neq 0 \\ 0 & \text { if } \xi=0\end{cases}
$$

Hence,

$$
A_{f}=\left\{\xi \in \mathbb{R}: \psi\left(\frac{1}{|\xi|^{\gamma}}\right)=a\right\} \cup\{0\} .
$$

Owing to the periodicity of $\psi, A_{f}$ has infinitely many bounded connected components. So, by Theorem 1.1, for every $M \geq 2$, there exists $\bar{\lambda}_{M}>0$ such 
that, for each $\lambda \in]-\bar{\lambda}_{M}, \bar{\lambda}_{M}[$, the above problem has at least $M+[M / 2]$ distinct solutions, $u_{i}^{(\lambda)}$ for $i=1, \ldots, M+[M / 2]$. Each solution $u_{i}^{(\lambda)}$ takes values in a bounded interval which does not depend on $\lambda \in]-\lambda_{M}, \lambda_{M}[$. The same stability property holds for their norms in $W^{1,2}(\Omega)$.

\section{References}

[1] G. Anello, A multiplicity theorem for critical points of functionals on reflexive Banach spaces, Arch. Math. (Basel) 82 (2004), 172-179.

[2] - Existence and multiplicity of solutions to a perturbed Neumann problem, Math. Nachr., to appear.

[3] D. Gilbarg and N. S. Trudinger, Elliptic Partial Differential Equations of Second Order, Springer, 2001.

[4] S. J. Li and Z. L. Liu, Perturbations from symmetric elliptic boundary value problems, J. Differential Equations 185 (2002), 271-280.

[5] Z. L. Liu and J. B. Su, Solutions of some nonlinear elliptic problems with perturbation terms of arbitrary growth, Discrete Contin. Dyn. Syst. 10 (2004), 617-634.

[6] P. Pucci and J. Serrin, A mountain pass theorem, J. Differential Equations 60 (1985), 142-149.

[7] B. Ricceri, A general variational principle and some of its applications, J. Comput. Appl. Math. 113 (2000), 401-410.

[8] - Sublevel sets and global minima of coercive functionals and local minima of their perturbations, J. Nonlinear Convex Anal. 5 (2004), 157-168.

[9] -, A multiplicity theorem for the Neumann problem, Proc. Amer. Math. Soc. 134 (2006), 1117-1124.

[10] C. G. Simader, Higher regularity of weak $L_{q}$-solutions of the Neumann problem for the Laplacian, Bayreuth. Math. Schr., in press.

[11] G. Stampacchia, Contributi alla regolarizzazione delle soluzioni dei problemi al contorno per equazioni del secondo ordine ellittiche, Ann. Scuola Norm. Sup. Pisa (3) 12 (1958), 223-245.

[12] E. Zeidler, Nonlinear Functional Analysis and its Applications, Vol. III, Springer, New York, 1984.

Department of Mathematics

University of Messina

98166 Sant'Agata-Messina, Italy

E-mail: cordaro@dipmat.unime.it

Received February 22, 2006

Revised version September 29, 2006 